\title{
Agencia de autocuidado de la persona con enfermedad renal crónica en diálisis
}

\author{
Claudia Patricia Cantillo-Medina, Joselinne de Jesús Blanco-0choa \\ Departamento de Enfermería. Facultad de Salud. Universidad Surcolombiana. Neiva. Huila. Colombia
}

\section{Introducción}

Las personas con Enfermedad Renal Crónica en diálisis permanentemente toman decisiones sobre lo que pueden y deben hacer en relación a su situación de salud, limitación y requerimiento personal para mantener la homeostasis y controlar los factores de riesgo; por lo tanto conocer y potenciar su capacidad de autocuidado es fundamental para diseñar estrategias que impacten sus necesidades.

Objetivo: Determinar la capacidad de autocuidado de las personas con Insuficiencia Renal Crónica en diálisis, así como su relación con las características sociodemográficas y clínicas.

Material y Método: Estudio cuantitativo descriptivo de corte transversal. La población estuvo conformada por 111 personas dializadas en una unidad renal de Florencia (Colombia). Se aplicaron los instrumentos: caracterización sociodemográfica y clínica, y capacidad de agencia de autocuidado en el paciente hipertenso. Los datos se analizaron mediante la estadística descriptiva.

Resultados: Edad promedio de la población 56 años (DE:+14); género masculino 55.9\%; su capacidad de agencia de autocuidado fue alta y media $(80,2 \%$ y 19,8\%) respectivamente; se encontró significación entre la variable capacidad de autocuidado y edad, que indica que a mayor edad, disminuye la capacidad de autocuidado $(p=0,024<0,05)$, además manifestaron mejor percepción del estado de salud aquellas personas con mayor grado de capacidad de agencia de autocuidado $(p=0,004<0,05)$.

\section{Correspondencia: \\ Claudia Patricia Cantillo-Medina \\ Avda. Pastrana Borrero, carrera $1^{a}$ \\ 410010 Neiva, Huila. Colombia \\ E-mail: claudiacantillo1@hotmail.com}

Conclusiones: La capacidad de agencia de autocuidado de las personas dializadas, se caracterizó por estar entre los niveles de alta y media capacidad, sin embargo se requiere de intervenciones del profesional de Enfermería y del equipo de salud, basadas en las necesidades individuales para mantener y mejorar su autocuidado.

PALABRAS CLAVE: autocuidado; atención de enfermería; enfermedad renal crónica; teoría de enfermería.

\section{Self-care agency in persons with chronic kidney disease in dialysis}

\begin{abstract}
People with Chronic Kidney Disease in dialysis permanently make decisions about what they can and should do in relation to their health situation, limitation and personal requirement to maintain homeostasis and control risk factors; therefore, knowing and strengthening their self-care capacity is fundamental to design strategies that impact their needs.
\end{abstract}

Objective: To determine the capacity of self-care of the people with Chronic Kidney Disease in dialysis, such as their relation with the sociodemographic and clinical characteristics.

Material y Method: A quantitative, descriptive, cross-sectional study. The population was formed by one hundred and eleven dialyzed people at the Medilaser Renal Unit in Florencia (Colombia). Sociodemographic and clinical variables and capacity of self-care agency in hypertensive patient were obtained. Data were analyzed through descriptive statistics. 
Resultados: Mean age of the population: fifty-six (56) years old $(S D \pm 14)$; male gender:

Fifty-five point nine per cent $(55.9 \%)$; their capacity of self-care agency was high and medium $(80,2 \%$ and $19,8 \%$, respectively). Significance was found between the self-care capacity variable and age, which indicates that higher age the self-care capacity decreases $(p=0.024)$, as well as a better perception of the state of health with greater capacity of people self-care agency $(p=0.004)$.

Conclusiones: The capacity of self-care agency of dialyzed people is between the high and medium capacity levels; however, it requires interventions from the nursing professional and health-care professionals, based on individual needs to maintain and improve their self-care.

KEYWORDS: self-care; nursing; chronic kidney disease; nursing theory.

\section{Introducción}

La Enfermedad Renal Crónica (ERC) es un problema de salud emergente a nivel mundial, forma parte del grupo de las enfermedades crónicas no transmisibles (ECNT) que están relacionadas con la forma de vivir de las personas, cuya incidencia y mortalidad aumenta anualmente de manera exponencial. Según la Organización Mundial de la Salud (OMS) las patologías crónicas demandan el $75 \%$ del gasto sanitario ${ }^{1}$ y con base en el elevado impacto biopsicosocial sobre la persona, su entorno familiar y social, se requieren criterios coordinados de los equipos de salud para garantizar la atención de la demanda con calidad².

Si la persona que vive con ERC además tiene factores de riesgo cardiovascular con comorbilidad asociada, presenta una supervivencia global de $12,9 \%$ a los diez años de permanencia en terapia de remplazo renal $(T R R)^{2}$. Desde esta perspectiva la ERC y las TRR per sé, implican riesgo de alteración en el autocuidado de la persona, acorde a las limitaciones que ésta genera y a la complejidad de los tratamientos.

El individuo inicia tratamiento con diálisis para restablecer y mantener la estabilidad de su organismo, controlar los síntomas urémicos y prolongar su vida dentro de los límites que admiten sus condiciones fí- sicas, psicológicas y sociales ${ }^{3}$. Teniendo en cuenta la complejidad del tratamiento dialítico y el impacto sobre la vida, es importante destacar que las terapias son efectivas y seguras cuando las personas realizan cambios en su estilo de vida y autocuidado como: adherencia al régimen terapéutico (alimentación, medicación), cuidado con el acceso para diálisis, actividad física, y manejo del estrés, entre otros aspectos; pues así contribuyen a controlar los factores de riesgo que pueden ocasionar complicaciones que afectan la calidad de vida ${ }^{3}$.

Enfermería debe brindar un cuidado en el cual se establezca una relación enfermera-paciente donde se reconozca al otro como un ser capaz de tomar sus propias decisiones y autodeterminarse, realizar las prácticas de autocuidado y mantener su autonomía, en aras de conservar una calidad de vida acorde con las limitaciones que pueda producir la enfermedad. En este sentido, el autocuidado es "Una función reguladora del hombre que las personas deben llevar a cabo deliberadamente por si solas, o deben de haber llevado a cabo para mantener su vida, salud, desarrollo y bienestar ${ }^{\prime \prime 4}$.

Por consiguiente, desde la disciplina de Enfermería es necesario fortalecer y generar nuevo conocimiento fundamentados en la Teoría del déficit de autocuidado de Orem, que permitan reconocer la capacidad de autocuidado de las personas con ERC en diálisis, aspecto importante para atender las necesidades de intervención y garantizar su gestión oportuna.

Por lo expuesto anteriormente, se plantea como objetivo determinar la capacidad de autocuidado de las personas con ERC en diálisis, así como su relación con las características sociodemográficas y clínicas.

\section{Material y Método}

Estudio cuantitativo descriptivo de corte transversal entre enero y junio de 2016. La población de estudio la conformaron el total de las personas con ERC en diálisis de una Unidad Renal en Florencia, Colombia $(n=111$, de las cuales 101 se encontraban en hemodiálisis -HD- y 10 en diálisis peritoneal -DP-), cumpliendo con los siguientes criterios de inclusión: mayor de 18 años, ser parte del programa de diálisis por un período mayor a tres meses, aceptación voluntaria a participar en el estudio. Se excluyeron las personas que presentaban diagnóstico de demencia o alteraciones cognitivas registradas en la historia clínica. 
Se recogieron las siguientes variables sociodemográficas: edad, género, escolaridad, estado civil, ocupación, régimen de convivencia, disponibilidad y tipo de cuidador, red de apoyo, procedencia, seguridad social y religión; y variables clínicas: antecedentes familiares, antecedentes personales, etiología de la ERC, tipo de tratamiento dialítico, tiempo en TRR y percepción del estado de salud (evaluado mediante una escala tipo Likert de 4 puntos - de malo a muy bueno-).

Asimismo, previa autorización de la autora, se empleó el Cuestionario de Capacidad de Agencia de Autocuidado en el Paciente Hipertenso (CCAAPH), elaborado por Achury et $\mathrm{al}^{5}$, constituido por 17 ítems que determinan la capacidad global de agencia de autocuidado. El instrumento tiene escala sumativa tipo Likert de 5 puntos (donde 1 es nunca y 5 siempre). Los rangos para evaluar el grado de capacidad de autocuidado se establecen en función la suma de puntos: Baja capacidad: 17-28, Media capacidad: 29-56 y Alta capacidad: 5785 , con una confiabilidad de 0,755 . El cuestionario fue autoadministrado.

Los datos se incorporaron en una matriz creada en Microsoft Excel 2016y se procesaron en el software estadístico IBMSPSS ${ }^{\circledR} 23$, con el cual se realizó un análisis descriptivo (frecuencias y porcentajes para las variables cualitativas, y medidas de tendencia central y de dispersión para las variables cuantitativas). Para identificar las variables asociadas al nivel de autocuidado, se comparó la distribución de las variables sociodemográficas y clínicas según el grado de capacidad de autocuidado, mediante la prueba de $\mathrm{Chi}^{2}$ o prueba exacta de Fisher. Además, se aplicó el test de Kruskal Wallis para comparar las variables ordinales y hallar si hubo diferencias entre el grupo de media capacidad y alta capacidad. En todos los casos se tuvo en cuenta un nivel de significación estadística cuando el valor $p<0,05$.

El proyecto fue aprobado por el Comité de Ética de la Clínica Medilaser Florencia, y se rigió bajo los principios éticos para la investigación con seres humanos definidos en la Resolución 008430 de 1993 que reglamenta la investigación en Colombia ${ }^{6}$, se tuvieron en cuenta los principios éticos: respeto a la dignidad humana, privacidad, libertad de expresión y sentimientos, confidencialidad y reciprocidad. Se solicitó el consentimiento informado para cada participante. El reclutamiento de pacientes se realizó dentro de la institución en el momento de asistencia a la consulta o al tratamiento dialítico, concretando el momento de aplicación de los instrumentos.

\section{Resultados}

\section{Características sociodemográficas y clínicas}

Obtuvimos una tasa de participación del 100\% $(n=111)$. El 49,5\% tenían 60 años o más, con un promedio de edad 56 años (DE:+14 años; rango 19 a 83 años). El perfil sociodemográfico de paciente de nuestra población fue: hombre, católico, casado o en unión libre, sin estudios o con estudios primarios, desempleados cobrando un subsidio, ejerciendo de cuidador su cónyuge, y disponiendo de red de apoyo social de tipo religioso. En la Tabla 1 se pueden observar las características sociodemográficas de la población a estudio, tanto para la totalidad, como en función de su capacidad de autocuidado.

En relación a las características clínicas de los participantes, el perfil se corresponde con una buena percepción su estado de salud como buena, sin antecedentes familiares relevantes, hipertenso, en tratamiento con hemodiálisis durante dos años o más. En la Tabla 2 se muestran las características clínicas de la población a estudio, tanto para la totalidad, como en función de su capacidad de autocuidado.

\section{Capacidad de Agencia de Autocuidado}

La capacidad global de agencia de autocuidado en la población de estudio según los rangos ponderales establecidos en el Cuestionario de Capacidad de Agencia de Autocuidado en el Paciente Hipertenso, fue valorada como alta $(\mathrm{N}=89,80,2 \%)$ y media $(\mathrm{N}=22,19,8 \%)$.

La población clasificada en la categoría alta capacidad de autocuidado, se caracterizó por ser en su mayoría hombres, edad entre 29 y 59 años, escolaridad primaria o ningún grado, con pareja (casados y en unión libre), desempleados, contaban con el cónyuge como cuidador, red de apoyo predominante el grupo religioso, seguridad social pertenecientes al régimen subsidiado y religión católica (Tabla 1).

En cuanto a las características relevantes en la población de media capacidad, que difería de los de alta capacidad, predominó el género femenino, edad 60 o más años y casados (Tabla 1).

Con respecto a la capacidad de autocuidado en asociación con las características sociodemográficas se identificó asociación estadísticamente significativa con la variable edad, que indica que a mayor edad, disminuye la capacidad de autocuidado $(p=0,024<0,05)$ ( Tabla 1$)$. 
Tabla 1. Capacidad de agencia de autocuidado en asociación con las características sociodemográficas de las personas en diálisis.

\begin{tabular}{|c|c|c|c|c|c|c|c|}
\hline \multirow{3}{*}{ Variable } & \multicolumn{4}{|c|}{ Capacidad de Autocuidado } & \multirow{3}{*}{$\mathbf{p}$} & \multirow{2}{*}{\multicolumn{2}{|c|}{$\begin{array}{c}\text { Total } \\
\text { Población }\end{array}$}} \\
\hline & \multicolumn{2}{|c|}{$\begin{array}{c}\text { Media } \\
\text { Capacidad }\end{array}$} & \multicolumn{2}{|c|}{$\begin{array}{c}\text { Alta } \\
\text { Capacidad }\end{array}$} & & & \\
\hline & $n$ & $\%$ & $\mathrm{n}$ & $\%$ & & $\mathrm{n}$ & $\%$ \\
\hline $\begin{array}{l}\text { Rangos de edad } \\
\text { 18-28 años } \\
29-59 \text { años } \\
\geq 60 \text { años }\end{array}$ & $\begin{array}{c}1 \\
5 \\
16 \\
\end{array}$ & $\begin{array}{c}4,5 \\
22,7 \\
72,7 \\
\end{array}$ & $\begin{array}{c}4 \\
46 \\
39 \\
\end{array}$ & $\begin{array}{r}4,5 \\
51,7 \\
43,8 \\
\end{array}$ & $\begin{array}{c}0,024^{*} \\
* *\end{array}$ & $\begin{array}{c}5 \\
51 \\
55\end{array}$ & $\begin{array}{r}4,5 \\
45,9 \\
49,5 \\
\end{array}$ \\
\hline $\begin{array}{l}\text { Género } \\
\text { Femenino } \\
\text { Masculino } \\
\end{array}$ & $\begin{array}{l}12 \\
10 \\
\end{array}$ & $\begin{array}{l}54,5 \\
45,5\end{array}$ & $\begin{array}{l}37 \\
52 \\
\end{array}$ & $\begin{array}{l}41,6 \\
58,4 \\
\end{array}$ & $0,273^{*}$ & $\begin{array}{l}49 \\
62 \\
\end{array}$ & $\begin{array}{l}44,1 \\
55,9 \\
\end{array}$ \\
\hline $\begin{array}{l}\text { Escolaridad } \\
\text { Ninguna-Primaria } \\
\text { Bachillerato } \\
\text { Postgrado } \\
\text { Técnico } \\
\text { Universitario } \\
\end{array}$ & $\begin{array}{l}19 \\
3 \\
0 \\
0 \\
0\end{array}$ & $\begin{array}{c}86,4 \\
13,6 \\
0 \\
0 \\
0\end{array}$ & $\begin{array}{c}56 \\
19 \\
2 \\
4 \\
8\end{array}$ & $\begin{array}{c}62,9 \\
21,3 \\
2,2 \\
4,5 \\
9\end{array}$ & $0,246^{*}$ & $\begin{array}{c}75 \\
22 \\
2 \\
4 \\
8\end{array}$ & $\begin{array}{c}67,6 \\
19,8 \\
1,8 \\
3,6 \\
7,2 \\
\end{array}$ \\
\hline $\begin{array}{l}\text { Estado Civil } \\
\text { Casado } \\
\text { Soltero } \\
\text { Unión Libre } \\
\text { Viudo } \\
\end{array}$ & $\begin{array}{c}10 \\
8 \\
0 \\
4\end{array}$ & $\begin{array}{c}45,5 \\
36,4 \\
0 \\
18,2\end{array}$ & $\begin{array}{c}33 \\
36 \\
13 \\
7\end{array}$ & $\begin{array}{c}37,1 \\
40,4 \\
14,6 \\
7,9 \\
\end{array}$ & $0,139 *$ & $\begin{array}{l}43 \\
44 \\
13 \\
11\end{array}$ & $\begin{array}{c}38,7 \\
39,6 \\
11,7 \\
9,9\end{array}$ \\
\hline $\begin{array}{l}\text { Ocupación } \\
\text { Desempleado } \\
\text { Empleado } \\
\text { Independiente } \\
\text { Jubilado }\end{array}$ & $\begin{array}{c}19 \\
0 \\
2 \\
1\end{array}$ & $\begin{array}{c}86,4 \\
0 \\
9,1 \\
4,5\end{array}$ & $\begin{array}{c}64 \\
5 \\
11 \\
9\end{array}$ & $\begin{array}{c}71,9 \\
5,6 \\
12,4 \\
10,1\end{array}$ & $0,476^{*}$ & $\begin{array}{c}83 \\
5 \\
13 \\
10\end{array}$ & $\begin{array}{c}74,8 \\
4,5 \\
11,7 \\
9 \\
\end{array}$ \\
\hline $\begin{array}{l}\text { Cuidador } \\
\text { Sin dato } \\
\text { Residencia geriátrica } \\
\text { Conyugue } \\
\text { Hermana } \\
\text { Hijo/a } \\
\text { Ninguno } \\
\text { Otro } \\
\text { Otro Familiar } \\
\text { Padre/Madre }\end{array}$ & $\begin{array}{l}1 \\
0 \\
0 \\
7 \\
0 \\
1 \\
2 \\
3\end{array}$ & $\begin{array}{c}4,5 \\
0 \\
36,4 \\
0 \\
31,8 \\
0 \\
4,5 \\
9,1 \\
13,6\end{array}$ & $\begin{array}{c}0 \\
1 \\
38 \\
1 \\
27 \\
2 \\
8 \\
3 \\
9\end{array}$ & $\begin{array}{c}0 \\
1,1 \\
42,7 \\
1,1 \\
30,3 \\
2,2 \\
9 \\
3,4 \\
10,1\end{array}$ & $0,603 *$ & $\begin{array}{c}1 \\
1 \\
46 \\
1 \\
34 \\
2 \\
9 \\
5 \\
12\end{array}$ & $\begin{array}{c}0,9 \\
0,9 \\
41,4 \\
0,9 \\
30,6 \\
1,8 \\
8,1 \\
4,5 \\
10,8\end{array}$ \\
\hline $\begin{array}{l}\text { Red de apoyo } \\
\text { Sin dato } \\
\text { Grupo de Amigos } \\
\text { Grupo Hospitalario } \\
\text { Grupo Religioso } \\
\text { Grupo Social } \\
\text { Otro }\end{array}$ & $\begin{array}{c}0 \\
3 \\
0 \\
17 \\
1 \\
1\end{array}$ & $\begin{array}{c}0 \\
13,6 \\
0 \\
77,3 \\
4,5 \\
4,5\end{array}$ & $\begin{array}{c}1 \\
15 \\
14 \\
58 \\
1 \\
0\end{array}$ & $\begin{array}{c}1,1 \\
16,9 \\
15,7 \\
65,2 \\
1,1 \\
0 \\
\end{array}$ & $0,094^{*}$ & $\begin{array}{c}1 \\
18 \\
14 \\
75 \\
2 \\
1\end{array}$ & $\begin{array}{c}0,9 \\
16,2 \\
12,6 \\
67,6 \\
1,8 \\
0,9 \\
\end{array}$ \\
\hline $\begin{array}{l}\text { Tipo de Seguridad social } \\
\text { Contributivo } \\
\text { Régimen especial } \\
\text { Subsidiado }\end{array}$ & $\begin{array}{c}3 \\
0 \\
19 \\
\end{array}$ & $\begin{array}{c}13,6 \\
0 \\
86,4 \\
\end{array}$ & $\begin{array}{c}28 \\
2 \\
59 \\
\end{array}$ & $\begin{array}{c}31,5 \\
2,2 \\
66,3\end{array}$ & $0,173^{*}$ & $\begin{array}{c}31 \\
2 \\
78\end{array}$ & $\begin{array}{c}27,9 \\
1,8 \\
70,3\end{array}$ \\
\hline $\begin{array}{l}\text { Religión practicada } \\
\text { Católica } \\
\text { Cristiana } \\
\text { Ninguna } \\
\text { Otra } \\
\text { Pentecostal }\end{array}$ & $\begin{array}{c}20 \\
0 \\
1 \\
0 \\
1\end{array}$ & $\begin{array}{c}90,9 \\
0 \\
4,5 \\
0 \\
4,5\end{array}$ & $\begin{array}{c}66 \\
16 \\
1 \\
4 \\
2\end{array}$ & $\begin{array}{c}74,2 \\
18 \\
1,1 \\
4,5 \\
2,2\end{array}$ & $0,132 *$ & $\begin{array}{c}86 \\
16 \\
2 \\
4 \\
3\end{array}$ & $\begin{array}{c}77,5 \\
14,4 \\
1,8 \\
3,6 \\
2,7\end{array}$ \\
\hline
\end{tabular}

${ }^{*}$ Chi2, **Prueba Exacta de Fisher, ***Kruskal-Wallis. 
Según el perfil clínico, la población con calificación alta de agencia de Autocuidado se caracterizó por: ser hipertensos, en tratamiento con hemodiálisis, tiempo mayor a dos años en TRR y buena percepción del estado de salud. En relación al nivel medio en la Agencia de Autocuidado, el perfil clínico se distinguió de los de nivel alto en que su estado de salud se percibía como "Regular". Se observó una correlación entre la percepción del estado de salud con la capacidad de agencia de autocuidado (para media $72,7 \%$ vs alta $56,2 \%$; $\mathrm{p}=0,004$ ) (Tabla 2).

Se observaron asociaciones significativas entre la capacidad de agencia de autocuidado de los participantes y los ítems $4,5,6,10,11,13$ y 14 del CCAAPH $(p<0,01)$; asimismo, se encontró asociación en los ítems 2,12 y $15(p<0,05)$. En estas preguntas los participantes con media capacidad puntuaron más bajo que los participantes con alta capacidad de autocuidado (Tabla 3).

\section{Discusión}

Las características sociodemográficas predominantes de las personas con ERC en diálisis fueron: edad 60 o más años; variable acorde con lo descrito por Méndez et $\mathrm{al}^{7}$, quienes explican el declive de la función renal como proceso fisiológico del envejecimiento desde la tercera década de la vida, deterioro que se acelera con la presencia de factores de riesgo asociados a estilos de vida inadecuados, por esta razón deben ser intervenidos desde temprana edad ${ }^{8,9,10}$. En relación al género la mayoría masculino $(55,9 \%)$, similar a lo descrito por Carrilo-Algarra y Díaz ${ }^{11}$; en estado civil sobresalieron las personas con pareja es decir: casados y en unión libre, la situación descrita se relaciona con el ciclo vital y familiar de los participantes ${ }^{12}$, este aspecto lo describe De la Cuesta ${ }^{13}$ como fundamental en el proceso de afrontamiento a la situación de salud, porque un vínculo de parentesco o afectivo-amoroso hace la diferencia entre el cuidado del familiar y el profesional.

El grado de escolaridad más frecuente fue primaria 0 ningún grado, cifras similares con otros estudios donde las personas con bajo nivel educativo por falta de conocimiento de la enfermedad y su manejo, presentaron un control inadecuado de patologías precursoras de $\mathrm{ERC}^{14,10}$; por consiguiente, es necesario fortalecer el conocimiento sobre el proceso salud-enfermedad y su manejo con intervenciones participativas, donde los individuos asuman el protagonismo, se incluyan sus percepciones, deseos y motivaciones en el proceso de toma de decisiones, para lograr empoderamiento y responsabilidad, en la medida en que las personas tienen mayor comprensión de su situación se espera un aumento de la capacidad para cuidar de sí mismos ${ }^{15}$.

La mayoría de los participantes estaban desempleados, condición que concuerda con los resultados encontrados por Julián-Mauro et $\mathrm{al}^{16}$, donde la ERC y las TRR son factores de incapacidad laboral, para lo cual recomiendan a las personas activas iniciar con terapias domiciliarias y luego continuar en los centros de diálisis a medida que se altere su autonomía o aumente la complejidad clínica.

El principal cuidador reportado fue el cónyuge o hijo, figura importante en el soporte emocional: $\operatorname{Costa}^{17}$ considera que la capacidad para desempeñar las actividades diarias se facilitan con la ayuda y el apoyo del compañero, pues contribuye a superar los obstáculos y desafíos impuestos por las limitaciones físicas ${ }^{13,18}$.

La mayoría de la población contaba con un grupo religioso como red de apoyo, según Silva ${ }^{19}$ es un aspecto favorable para el afrontamiento: esto crea un efecto protector, mejor tratamiento, adherencia a la medicación y nutrición, mejores resultados clínicos, que aumentan la satisfacción y calidad de vida relacionada con la salud.

En lo que se refiere a las características clínicas se encontró un alto porcentaje de la población con etiología de hipertensión arterial, similar con los hallazgos reportados por varios autores 8 -11,14,20; quiénes plantean que un bajo nivel socioeconómico y educativo se asocian al control inadecuado de patologías, especialmente hipertensión arterial y diabetes mellitus: posiblemente porque no se comprende el proceso de la enfermedad, manejo adecuado con la dieta, medicamentos o ausencia de recursos para asistir a la consulta. Se resalta que las personas con hipertensión arterial, el $42.3 \%$ no logran el control, condición responsable del $62 \%$ de casos de enfermedad cerebrovascular y $49 \%$ de enfermedad cardiaca isquémica ${ }^{21}$, por esta razón la 0MS la reporta como factor de riesgo importante para mortalidad a nivel mundial ${ }^{22}$.

Se halló gran proporción de personas en tratamiento con permanencia mayor a dos años; con percepción sobre su estado de salud como bueno a pesar de vivir en condición de cronicidad, aspecto coherente con lo descrito por Achury ${ }^{23}$, al referir que la persona con el tiempo y trayectoria de vida aprende a convivir con una enfermedad crónica y ciertos padecimientos. 
Tabla 2. Capacidad de agencia de autocuidado en asociación con las características clínicas de las personas dializadas.

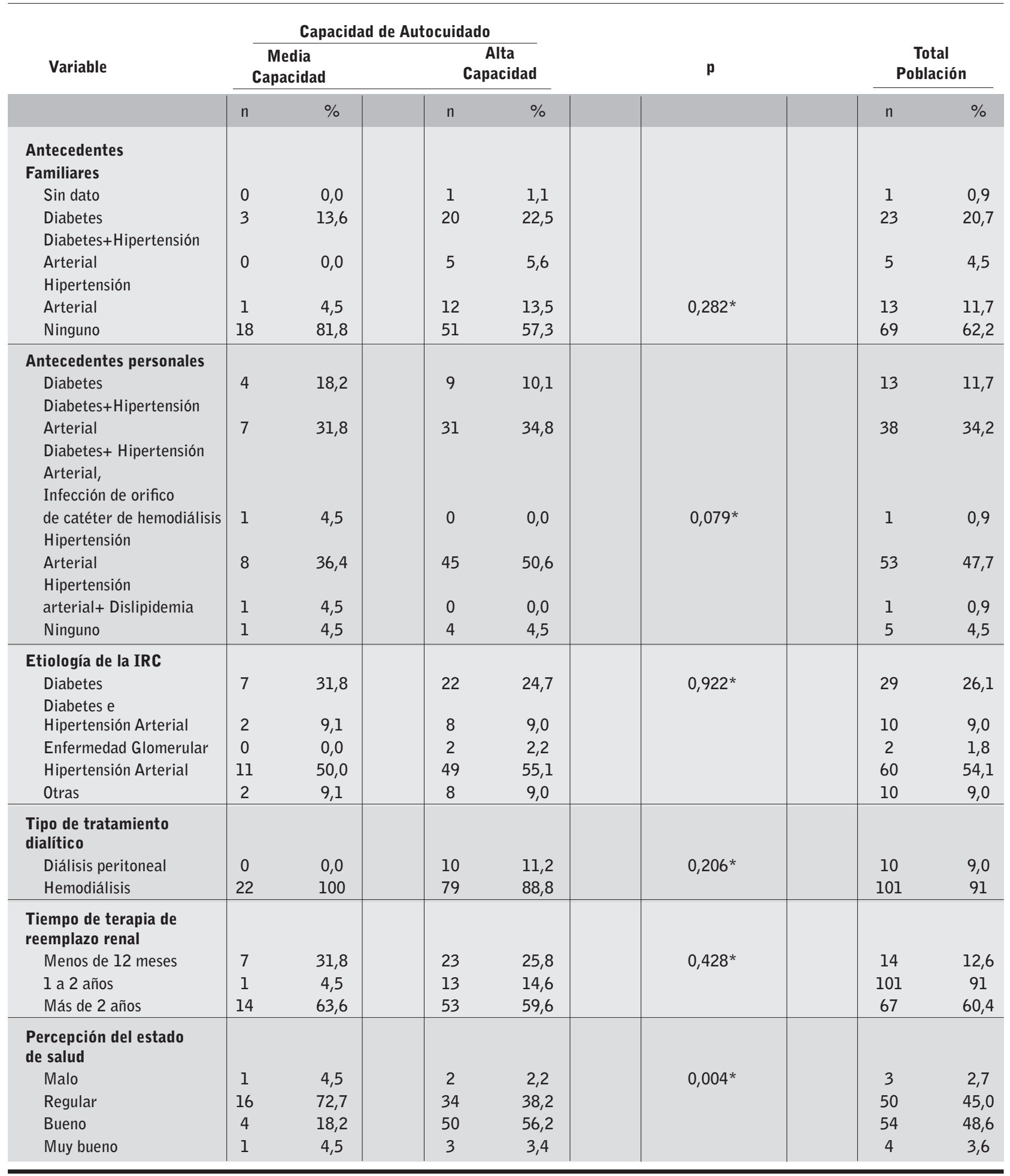

*Kruskal-Wallis. 
Tabla 3. Asociación entre los ítems y la valoración global de la capacidad de agencia de Autocuidado.

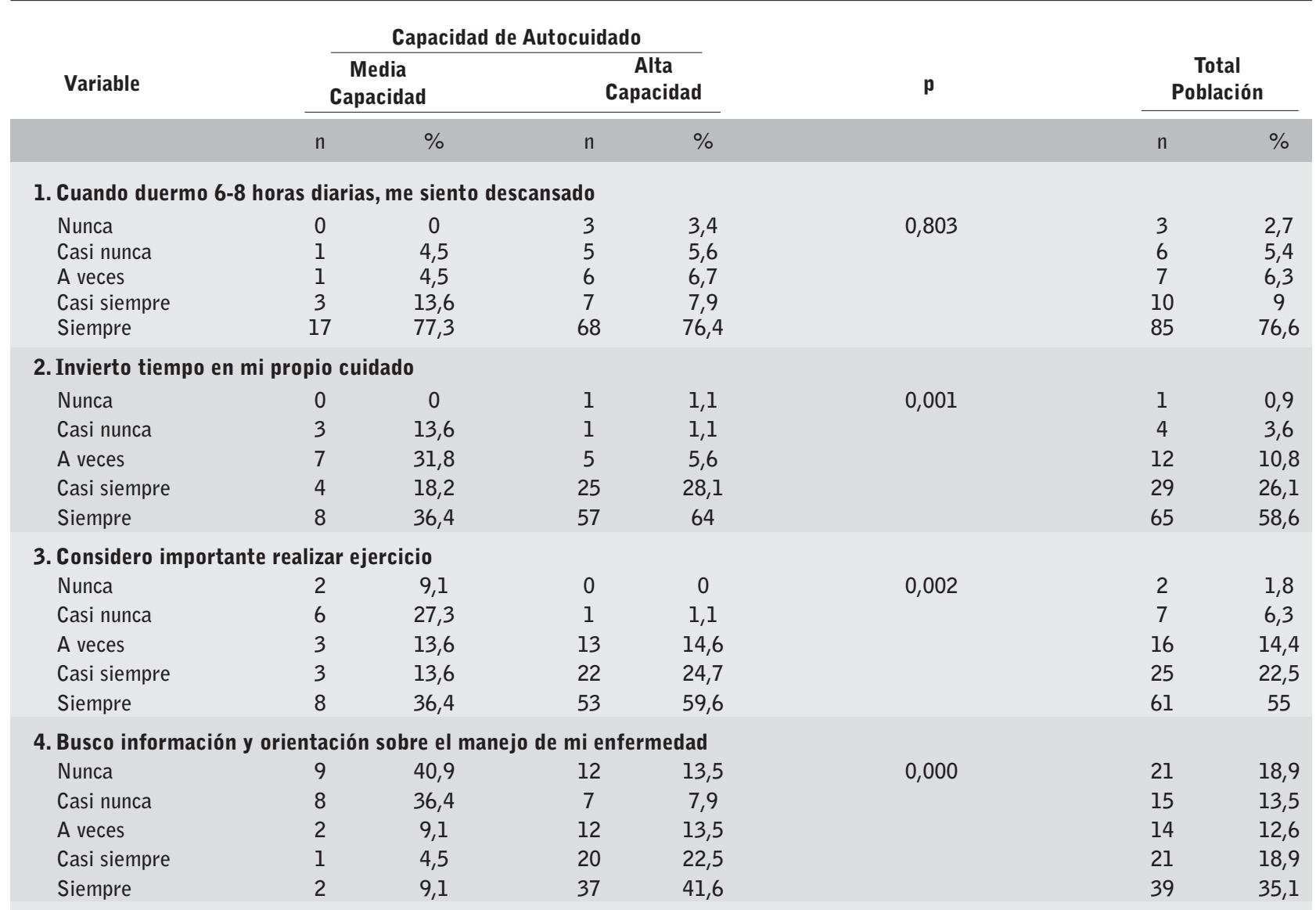

5. Considero que conozco como actúan y las reacciones desfavorables de los medicamentos que tomo actualmente para controlar la presión arterial

\begin{tabular}{|c|c|c|c|c|c|c|c|}
\hline Nunca & 11 & 50 & 22 & 24,7 & 0,000 & 33 & 29,7 \\
\hline Casi nunca & 8 & 36,4 & 8 & 9 & & 16 & 14,4 \\
\hline$A$ veces & 1 & 4,5 & 18 & 20,2 & & 19 & 17,1 \\
\hline Casi siempre & 1 & 4,5 & 12 & 13,5 & & 13 & 11,7 \\
\hline Siempre & 1 & 4,5 & 29 & 32,6 & & 30 & 27 \\
\hline
\end{tabular}

6. Cuando inicio un nuevo medicamento solicito al profesional de enfermería me proporcione información necesaria de la correcta administración

\begin{tabular}{|c|c|c|c|c|c|c|c|}
\hline Nunca & 6 & 27,3 & 8 & 9 & 0,000 & 14 & 12,6 \\
\hline Casi nunca & 5 & 22,7 & 3 & 3,4 & & 8 & 7,2 \\
\hline$A$ veces & 3 & 13,6 & 6 & 6,7 & & 9 & 8,1 \\
\hline Casi siempre & 3 & 13,6 & 22 & 24,7 & & 25 & 22,5 \\
\hline Siempre & 5 & 22,7 & 50 & 56,2 & & 5 & 49,5 \\
\hline
\end{tabular}

7. Considero que la cantidad máxima de sal que puedo utilizar para todo el día en la preparación y consumo de alimentos son 2 cucharaditas de sal

$\begin{array}{lccccccc}\text { Nunca } & 0 & 0 & 2 & 2,2 & 0,960 & 2 & 1,8 \\ \text { Casi nunca } & 0 & 0 & 0 & 0 & & 0 & 7 \\ \text { A veces } & 3 & 13,6 & 4 & 4,5 & & 17 & 15,3 \\ \text { Casi siempre } & 2 & 9,1 & 15 & 16,9 & & 85 & 76,6 \\ \text { Siempre } & 17 & 77,3 & 68 & 76,4 & & \\ \text { Reemplazo mi tratamient } & \text { por terapias alternativas } & & & 0,351 & 94 & 84,7 \\ \text { Nunca } & 20 & 90,9 & 74 & 83,1 & & 3 & 2,7 \\ \text { Casi nunca } & 0 & 0 & 3 & 3,4 & & 8 & 7,2 \\ \text { A veces } & 2 & 9,1 & 6 & 6,7 & & 3 & 2,7 \\ \text { Casi siempre } & 0 & 0 & 3 & 3,4 & & 3 & 2,7 \\ \text { Siempre } & 0 & 0 & 3 & 3,4 & & & \\ \end{array}$


Tabla 3. Asociación entre los ítems y la valoración global de la capacidad de agencia de Autocuidado. (continuación).

\begin{tabular}{|c|c|c|c|c|c|c|c|}
\hline \multirow{3}{*}{ Variable } & \multicolumn{4}{|c|}{ Capacidad de Autocuidado } & \multirow{3}{*}{$\mathbf{p}$} & \multirow{2}{*}{\multicolumn{2}{|c|}{$\begin{array}{c}\text { Total } \\
\text { Población }\end{array}$}} \\
\hline & \multicolumn{2}{|c|}{$\begin{array}{c}\text { Media } \\
\text { Capacidad }\end{array}$} & \multicolumn{2}{|c|}{$\begin{array}{c}\text { Alta } \\
\text { Capacidad }\end{array}$} & & & \\
\hline & $\mathrm{n}$ & $\%$ & $\mathrm{n}$ & $\%$ & & $\mathrm{n}$ & $\%$ \\
\hline \multicolumn{8}{|c|}{ 9. Considero que el profesional de enfermería me escucha y aclara mis inquietudes } \\
\hline $\begin{array}{l}\text { Nunca } \\
\text { Casi nunca } \\
\text { A veces } \\
\text { Casi siempre } \\
\text { Siempre }\end{array}$ & $\begin{array}{c}0 \\
0 \\
6 \\
4 \\
12\end{array}$ & $\begin{array}{c}0 \\
0 \\
27,3 \\
18,2 \\
54,5\end{array}$ & $\begin{array}{c}1 \\
0 \\
8 \\
18 \\
62\end{array}$ & $\begin{array}{c}1,1 \\
0 \\
9 \\
20,2 \\
69,7\end{array}$ & 0,108 & $\begin{array}{c}1 \\
0 \\
14 \\
22 \\
74\end{array}$ & $\begin{array}{c}0,9 \\
0 \\
12,6 \\
19,8 \\
66,7\end{array}$ \\
\hline \multicolumn{8}{|c|}{ 10. Conozco las complicaciones derivadas de la enfermedad } \\
\hline $\begin{array}{l}\text { Nunca } \\
\text { Casi nunca } \\
\text { A veces } \\
\text { Casi siempre } \\
\text { Siempre }\end{array}$ & $\begin{array}{l}9 \\
8 \\
2 \\
1 \\
2\end{array}$ & $\begin{array}{c}40,9 \\
36,4 \\
9,1 \\
4,5 \\
9,1\end{array}$ & $\begin{array}{c}9 \\
18 \\
13 \\
8 \\
41\end{array}$ & $\begin{array}{c}10,1 \\
20,2 \\
14,6 \\
9 \\
46,1\end{array}$ & $\begin{array}{c}9 \\
43\end{array}$ & $\begin{array}{c}18 \\
26 \\
15 \\
8,1 \\
38,7\end{array}$ & $\begin{array}{l}16,2 \\
23,4 \\
13,5\end{array}$ \\
\hline \multicolumn{8}{|c|}{ 11. Camino diariamente como mínimo 30 minutos } \\
\hline $\begin{array}{l}\text { Nunca } \\
\text { Casi nunca } \\
\text { A veces } \\
\text { Casi siempre } \\
\text { Siempre }\end{array}$ & $\begin{array}{c}16 \\
4 \\
0 \\
0 \\
1\end{array}$ & $\begin{array}{c}72,7 \\
18,2 \\
0 \\
0 \\
4,5\end{array}$ & $\begin{array}{c}20 \\
8 \\
17 \\
8 \\
33\end{array}$ & $\begin{array}{c}22,5 \\
9 \\
19,1 \\
9 \\
37,1\end{array}$ & 0,000 & $\begin{array}{c}36 \\
12 \\
17 \\
8 \\
34\end{array}$ & $\begin{array}{r}32,4 \\
10,8 \\
15,3 \\
7,2 \\
30,6\end{array}$ \\
\hline \multicolumn{8}{|c|}{ 12. Realizo cambios según las necesidades, para mantener la salud y manejar adecuadamente la enfermedad. } \\
\hline $\begin{array}{l}\text { Nunca } \\
\text { Casi nunca } \\
\text { A veces } \\
\text { Casi siempre } \\
\text { Siempre }\end{array}$ & $\begin{array}{l}0 \\
6 \\
7 \\
3 \\
6\end{array}$ & $\begin{array}{c}0 \\
27,3 \\
31,8 \\
13,6 \\
27,3\end{array}$ & $\begin{array}{c}0 \\
2 \\
15 \\
25 \\
46\end{array}$ & $\begin{array}{c}0 \\
2,2 \\
16,9 \\
28,1 \\
51,7\end{array}$ & 0,001 & $\begin{array}{c}0 \\
8 \\
22 \\
28 \\
52\end{array}$ & $\begin{array}{c}0 \\
7,2 \\
19,8 \\
25,2 \\
46,8\end{array}$ \\
\hline \multicolumn{8}{|c|}{ 13. Realizo cambios en mi dieta para mantener un peso adecuado } \\
\hline $\begin{array}{l}\text { Nunca } \\
\text { Casi nunca } \\
\text { A veces } \\
\text { Casi siempre } \\
\text { Siempre }\end{array}$ & $\begin{array}{c}3 \\
6 \\
11 \\
2 \\
0\end{array}$ & $\begin{array}{c}13,6 \\
27,3 \\
50 \\
9,1 \\
0\end{array}$ & $\begin{array}{c}3 \\
2 \\
24 \\
24 \\
36\end{array}$ & $\begin{array}{c}3,4 \\
2,2 \\
27 \\
27 \\
40,4\end{array}$ & 0,000 & $\begin{array}{c}6 \\
8 \\
35 \\
26 \\
36\end{array}$ & $\begin{array}{c}5,4 \\
7,2 \\
31,5 \\
23,4 \\
32,4\end{array}$ \\
\hline \multicolumn{8}{|c|}{ 14. Evalúo si las medidas que he tomado en el manejo de mi enfermedad permiten garantizar mi bienestar y el de mi familia } \\
\hline $\begin{array}{l}\text { Nunca } \\
\text { Casi nunca } \\
\text { A veces } \\
\text { Casi siempre } \\
\text { Siempre }\end{array}$ & $\begin{array}{l}3 \\
1 \\
9 \\
8 \\
1\end{array}$ & $\begin{array}{c}13,6 \\
4,5 \\
40,9 \\
36,4 \\
4,5\end{array}$ & $\begin{array}{c}1 \\
1 \\
6 \\
23 \\
58\end{array}$ & $\begin{array}{r}1,1 \\
1,1 \\
6,7 \\
25,8 \\
65,2\end{array}$ & 0,000 & $\begin{array}{c}4 \\
2 \\
15 \\
31 \\
59\end{array}$ & $\begin{array}{r}3,6 \\
1,8 \\
13,5 \\
27,9 \\
53,2\end{array}$ \\
\hline \multicolumn{8}{|c|}{ 15. Consumo en mi dieta comidas ricas en verduras, frutas, fibra y baja en grasas } \\
\hline $\begin{array}{l}\text { Nunca } \\
\text { Casi nunca } \\
\text { A veces } \\
\text { Casi siempre } \\
\text { Siempre }\end{array}$ & $\begin{array}{c}1 \\
2 \\
11 \\
6 \\
2\end{array}$ & $\begin{array}{c}4,5 \\
9,1 \\
50 \\
27,3 \\
9,1\end{array}$ & $\begin{array}{c}1 \\
3 \\
27 \\
13 \\
45\end{array}$ & $\begin{array}{c}1,1 \\
3,4 \\
30,3 \\
14,6 \\
50,6\end{array}$ & $\begin{array}{l}19 \\
47\end{array}$ & $\begin{array}{c}2 \\
5 \\
38 \\
17,1 \\
42,3\end{array}$ & $\begin{array}{r}1,8 \\
4,5 \\
34,2\end{array}$ \\
\hline \multicolumn{8}{|c|}{ 16. He tenido dificultades con el acceso a las consultas, controles y adquisición de medicamentos } \\
\hline $\begin{array}{l}\text { Nunca } \\
\text { Casi nunca } \\
\text { A veces } \\
\text { 6Casi siempre } \\
\text { Siempre }\end{array}$ & $\begin{array}{l}7 \\
2 \\
2 \\
6 \\
5\end{array}$ & $\begin{array}{c}31,8 \\
9,1 \\
9,1 \\
27,3 \\
22,7\end{array}$ & $\begin{array}{c}29 \\
3 \\
12 \\
17 \\
28\end{array}$ & $\begin{array}{c}32,6 \\
3,4 \\
13,5 \\
19,1 \\
31,5\end{array}$ & 0,698 & $\begin{array}{c}36 \\
5 \\
14 \\
23 \\
33\end{array}$ & $\begin{array}{c}32,4 \\
4,5 \\
12,6 \\
20,7 \\
29,7\end{array}$ \\
\hline \multicolumn{8}{|c|}{ 17. He tenido dificultades con el acceso a las consultas, controles y adquisición de medicamentos } \\
\hline $\begin{array}{l}\text { Nunca } \\
\text { Casi nunca } \\
\text { A veces } \\
\text { Casi siempre } \\
\text { Siempre }\end{array}$ & $\begin{array}{c}2 \\
1 \\
5 \\
2 \\
11\end{array}$ & $\begin{array}{c}9,1 \\
4,5 \\
22,7 \\
9,1 \\
50\end{array}$ & $\begin{array}{c}18 \\
0 \\
10 \\
5 \\
55\end{array}$ & $\begin{array}{c}20,2 \\
0 \\
11,2 \\
5,6 \\
61,8\end{array}$ & 0,684 & $\begin{array}{c}20 \\
1 \\
15 \\
7 \\
66\end{array}$ & $\begin{array}{c}18 \\
0,9 \\
13,5 \\
6,3 \\
59,5\end{array}$ \\
\hline
\end{tabular}

${ }^{*}$ Chi2, ${ }^{* *}$ Prueba Exacta de Fisher, ${ }^{* * *}$ Kruskal-Wallis. 
En relación a la capacidad global de agencia de autocuidado en la población de estudio se reportaron predominantemente con alta capacidad y en menor proporción con media capacidad, resultados similares a los hallazgos encontrados por otros estudios ${ }^{10,11,24}$. Como resultado de la asociación entre las características sociodemográficas y clínicas de las personas en diálisis con la capacidad de agencia de autocuidado, se encontró significación en las variables edad y percepción del estado de salud.

Con el progreso de la edad y la enfermedad crónica se produce deterioro funcional de la persona, y riesgo de disminuir el grado de capacidad de agencia de autocuidado ${ }^{8}$ : al disminuir éste se incrementa el riesgo de morbilidad, produciendo cambios en la percepción del estado de salud; de una percepción buena en las personas con alta capacidad, pasan a regular en aquellas con media capacidad.

Según los planteamientos de Orem, la edad, el estado de desarrollo y el estado de salud ${ }^{4}$; son factores básicos que condicionan o alteran la actividad de autocuidado de un individuo en momentos concretos y bajo circunstancias específicas. Fernández et $\mathrm{al}^{25}$ y Rodríguez et $\mathrm{al}^{26}$ partiendo de la propuesta de esta teórica, describen que la edad, el estado de desarrollo, el estado de salud, los factores del ambiente y los patrones de vida, tienen una asociación fuerte con la capacidad de autocuidado. Lo anteriormente descrito se corrobora en los hallazgos de esta investigación.

Con respecto al tratamiento dialítico es de resaltar que el total de la población en DP tiene alta capacidad de autocuidado, situación que favorece el éxito del tratamiento pues este se encuentra ligado a las prácticas de autocuidado: dieta, medicamentos, terapia de remplazo renal, cuidado con el acceso para diálisis ${ }^{27}$; aspectos importantes porque ellos mismos realizan el procedimiento de diálisis y viven su cotidianidad sin acompañamiento directo del equipo de salud.

En cuanto al tiempo de permanencia en TRR, predominó la población con un tiempo mayor de dos años, de la cual el mayor porcentaje presentó media capacidad de autocuidado: las personas cuando viven en situación de cronicidad aprenden a convivir con ciertos padecimientos, condición que puede influir en no seguir con los tratamientos ni cuidados recomendados ${ }^{15}$.

Según el análisis de los resultados de la relación de los ítems del CCAAPH con el grado de capacidad de las personas en diálisis, se identificaron aspectos re- levantes de conocimiento y habilidades que necesitan ser intervenidos para potenciar el autocuidado, como son: conocimiento del manejo y complicaciones de la enfermedad; conocimiento y manejo de medicamentos; conocimiento y habilidad dieta; actividad física y ejercicio; y evaluación de las medidas tomadas para mantener el bienestar.

Estos aspectos descritos anteriormente representan una oportunidad para incidir en los factores de riesgo para este grupo, los programas de educación para el control de enfermedades crónicas se constituyen en una directriz para mejorar la calidad de vida y reducir complicaciones ${ }^{28}$.

Aunque la mayoría de la población en diálisis presentó una alta capacidad de agencia de autocuidado, se evidenció que posee habilidades para identificar condiciones y situaciones que deben controlar en su día a día; sin embargo, como lo plantea Bonilla29: "La educación sanitaria es una herramienta de suma importancia en el tratamiento de los pacientes con ERC avanzada, y se debe ofrecer en el marco de una consulta formada por un equipo multidisciplinar, en el que Enfermería tiene un rol activo muy importante", debe ser regular, participativa y evaluada de manera continua.

En este sentido es necesario realizar la planificación de los cuidados de Enfermería, teniendo en cuenta los requerimientos individuales, las diversas dimensiones del ser humano y sus contextos, como lo expresa Mesa et al30: "La dimensión subjetiva y experiencial resultan determinantes en el afrontamiento de los procesos

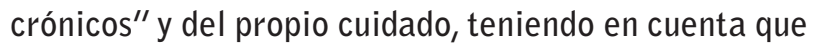
la edad, el estado de desarrollo y el estado de salud fueron factores condicionantes que se encontraron asociados a la capacidad de agencia de autocuidado en esta población.

Los resultados de esta investigación son útiles para avanzar en el campo de la investigación, cualificar la atención de Enfermería en el proceso de potenciación del autocuidado de las personas en diálisis; teniendo en cuenta la condición de vivir con una enfermedad progresiva, no curable y con tratamientos de alta complejidad que para garantizar su efectividad y mejorar su bienestar deben asumir cambios en los estilos de vida y con ellos grandes restricciones como lo describe Orem $^{31}$, Carrillo et $\mathrm{a}^{32}$ y Jiménez et $\mathrm{al}^{33}$. 


\section{Conclusiones}

La capacidad de agencia de autocuidado de las personas en diálisis se caracterizó por estar entre los niveles de alta y media capacidad, se evidenció relación de la capacidad de autocuidado con las variables edad y percepción del estado de salud; ratificando lo planteado sobre los factores básicos que condicionan o alteran la actividad de autocuidado de un individuo.

Aunque, la mayoría de la población presentó una alta capacidad de autocuidado es fundamental la intervención del equipo de salud, especialmente Enfermería, en relación a los aspectos de conocimiento y habilidad que impactan en el grado de capacidad de agencia de autocuidado, en aras de fortalecer, mantener y potenciar dicha capacidad, que los lleven a cambios en el comportamiento y conductas permanentes para disminuir su morbimortalidad, teniendo en cuenta que la población es cada vez más adulta y su proceso de salud más vulnerable.

Sobre la base de las consideraciones anteriores, resulta oportuno plantear intervenciones efectivas que sean soporte a largo plazo para que las personas con capacidad de agencia de autocuidado media, progresen a alta capacidad a través del empoderamiento del individuo junto con sus familias, para así impactar en la morbilidad y mejorar la calidad de vida.

\section{Limitaciones}

La limitación de esta investigación fue el empleo de un muestreo por conveniencia, situación que no hace posible la generalización de los resultados de la investigación. De igual manera el número de participantes en tratamiento de diálisis peritoneal, que es bajo en comparación con las personas en tratamiento de Hemodiálisis.

\section{Financiación}

Las autoras recibieron financiación para el desarrollo de la investigación de la Vicerrectoría de investigación y proyección social de la Universidad Surcolombiana.

\section{Agradecimientos}

Agradecemos a las personas con ERC en tratamiento de diálisis por su participación en este proyecto, a la Clínica Medilaser Florencia y a la Universidad Surcolombiana por el apoyo recibido.
El autor declara que no hay conflicto de interés.

Recibido: 25 octubre 2017

Revisado: 5 noviembre 2017

Modificado: 10 noviembre 2017

Aceptado: 12 noviembre 2017

\section{Bibliografía}

1. Organización Mundial de la Salud. Global status report on noncommunicable diseases 2014. Switzerland: Organización Mundial de la Salud; 2014.

2. Vargas Marcos F. Documento Marco sobre Enfermedad Renal Crónica (ERC) dentro de la Estrategia de Abordaje a la Cronicidad en el SNS. Madrid: Ministerio de Sanidad Servicios Sociales e Igualdad; 2015.

3. Trentini, M, Corradi, EM, Araldi Aparecida Raposo, M, Tigrinho Camila, F. Qualidade de vida de pessoas dependentes de hemodiálise considerando alguns aspectos físicos, sociais e emocionais. Texto \& Contexto Enfermagem 2004;13(1):74-82.

4. Hardin SR, Bishop SM. Unidad 1: Evolución de las teorías en Enfermería. Historia y filosofía de la ciencia. En: Raile Alligood M, Marriner Tomey A. Modelos y teorías en enfermería. $7^{a}$ Edición. Barcelona: Elsevier;2011. p. 16-25.

5. Achury Beltrán LF, Achury Saldaña DM, Rodríguez Colmenares SM, Sepúlveda Carrillo GJ, Padilla Velasco MP, et al. Capacidad de agencia de autocuidado en el paciente con hipertensión arterial en una institución de segundo nivel. Investig. Enferm. Imagen Desarr. 2012; 14 (2): 61-83.

6. Ministerio de Salud, Republica de Colombia. Resolución No 008430 de 1993. Por lo cual se establecen las normas científicas, técnicas y administrativas para la investigación en salud. Congreso de la Republica. Bogotá D.C. 1993.

7. Méndez-Durán A, Pérez-Aguilar G, Ayala-Ayala $F$, Ruiz-Rosas RA, González- Izquierdo JJ, Dávila-Torres J. Panorama epidemiológico de la insuficiencia renal crónica en el segundo nivel de atención del Instituto Mexicano del Seguro Social. Dial Traspl. 2014; 35:148-56. 
8. Martínez ME, Plazas $M$, Barajas GP, Bravo AM, González C, Rodríguez A, et al. Factores de riesgo para enfermedadrenal crónica en pacientes que asisten a consulta de medicina interna. Acta Médica Colomb. 2013;38(4):228-32.

9. Gámez Jiménez Ana Margarita, Montell Hernández Oscar Antonio, Ruano Quintero Vivian, Alfonso de León José A, Hay de la Puente Zoto Marlen. Enfermedad renal crónica en el adulto mayor. Rev. Med. Electrón. 2013; 35(4): 306-318.

10. Carrillo Algarra AJ. Análisis de la capacidad de autocuidados en pacientes en diálisis peritoneal. Enferm Nefrol. 2015; 18 (1): 31-40.

11. Carrillo Algarra AJ, Díaz FJ. Capacidad de autocuidado de los pacientes en diálisis peritoneal: un estudio piloto en Bogotá. Enferm glob. 2013; 12(30): 54-64.

12. Semenova Moratto Vásquez, N, Zapata Posada, JJ, Messager, T. Conceptualización de ciclo vital familiar: una mirada a la producción durante el periodo comprendido entre los años 2002 a 2015. CES Psicología. 2015;8(2):103-121.

13. De la Cuesta C. Cuidado Familiar en Condiciones Crónicas: Una Aproximación a la Literatura. Texto Context Enfermería. 2004;13(1):137-46.

14. Plantinga LC, Johansen $K L$, Schillinger $D$, Powe NR. Lower socioeconomic status and disability among US adults with chronic kidney disease, 1999-2008. Prev Chronic Dis. 2012;9:E12.

15. Pérez Acuña $C$, Riquelme Hernández G, Scharager Goldenberg J, Armijo Rodríguez I. Relación entre calidad de vida y representación de enfermedad en personas con enfermedad renal crónica terminal en tratamiento con hemodiálisis. Enferm Nefrol 2015; 18 (2): 89-96.

16. Julián-Mauro JC, Molinuevo-Tobalina JÁ, Sánchez-González JC. La situación laboral del paciente con enfermedad renal crónica en función del tratamiento sustitutivo renal. Nefrologia 2012;32(4):439-45.

17. Costa GMA, Pinheiro MBGN, Medeiros SM, Costa RRO, Cossi MS. Calidad de vida en pacientes con insuficiencia renal crónica en hemodiálisis. Enferm. glob. 2016; 15(43): 59-73.
18. Burgos Jiménez $E$, Meléndez Balderrama MA., Meza Coronado E, Agramón Cota KG, Pereyra Hernández $\mathrm{MC}$, et al. Impacto de una intervención dirigida a incrementar el conocimiento de la enfermedad renal sobre el inicio oportuno de la terapia sustitutiva. Rev Soc Esp Enferm Nefrol 2011; 14(4): 236-241.

19. Silva SM, Braido NF, Ottaviani AC, Gesualdo GD, Zazzetta MS, Orlandi F de S.Social support of adults and elderly with chronic kidney disease on dialysis. RevLat Am Enfermagem. 2016 Aug 8;24:e2752.

20. Pérez-Durillo $F T$, Villarejo-Villar $A B$, PérezDurillo J, Ribes-Bautista AI, Macías- Ortiz de Galisteo C. Enfermedad renal oculta a través de las ecuaciones de filtrado glomerular en Atención Primaria. Nefrologia 2014;34(5):676-8.

21. Appel LJ, Wright JT Jr, Greene T, Agodoa LY, Astor BC, Bakris GL, et al; AASK Collaborative Research Group. Intensive blood-pressure control in hypertensive chronic kidney disease. $\mathrm{N}$ Engl J Med. 2010 Sep 2;363(10):918-29.

22. World Health Organization. Global Database on Body Mass Index. [Internet].Switzerland: World Health Organization; 2014. [Consultado 10 diciembre 2016].Disponible en: http://apps.who. int/bmi/index.jsp?introPage=intro_3.html:bmi

23. Achury Saldaña DM. Autocuidado y adherencia en pacientes con falla cardiaca.Aquichan.2007;7(2):139-60.

24. Leiva Díaz V, Acosta Rojas P, Berrocal Barboza Y, Carrillo Sancho E, Castro Marín M. Capacidad de agencia de autocuidado en las personas adultas que padecen artritis reumatoide. Rev Enfermería actual en Costa Rica. 2012;22: 1-11.

25. Fernández AR, Manrrique FG. Agencia de autocuidado y factores básicos condicionantes en adultos mayores. Av. Enferm 2011; 29(1): 30-41.

26. Rodríguez Gázquez MA, Arredondo Holguín E, Salamanca Acevedo YA. Capacidad de agencia de autocuidado y factores relacionados con la agencia en personas con insuficiencia cardíaca de la ciudad de Medellín (Colombia). Enferm. glob. 2013;12(30):183-195. 
27. Sellarés VL, Torres Ramírez A, Hernández Merrero D. Manual de nefrología clínica, diálisis y trasplante renal. Madrid: Elsevier; 1997.

28. Jayasinghe J. Non-adherence in the hypertensive patient: can nursing play a role in assessing and improving compliance? Can J Cardiovasc Nurs. 2009;19(1):7-12.

29. Bonilla León FJ. Educación sanitaria al paciente con enfermedad renal crónica avanzada. ¿Existe evidencia de su utilidad? Enferm Nefrol 2014;17(2):120-31.

30. Mesa Melgarejo L, Carrillo Algarra AJ, Moreno Rubio F. La cronicidad y sus matices: estudio documental. Investig en Enfermería Imagen y Desarro. 2013;15(2):95-114.
31. Orem D. Normas prácticas en enfermería. Traducido por Susan Allen. Madrid, España: Ediciones Pirámide; 1983.

32. Carrillo Algarra AJ, Laza Vásquez $C$, Molina Jerena JÁ, Julia A, Algarra C, Vásquez CL, et al. Estudio documental ( 2006-2013 ) sobre el autocuidado en el día a día del paciente con enfermedad renal crónica. Enferm Nefrol 2013;16(3):185-192.

33. Jiménez AJ, Simón $V E$, Pinedo GI, Bernabeú ET, Soler IL, López OP, et al. Resultados de un programa de ejercicio físico combinado con electroestimulación neuromuscular en pacientes en hemodiálisis. Enferm Nefrol 2013;16(3):161-7.

Este artículo se distribuye bajo una Licencia Creative Commons Atribución-NoComercial 4.0 Internacional. https://creativecommons.org/licenses/by-nc/4.0/

\section{Open Access (C) (1) (8)}

